\title{
De novo mutation and skewed X-inactivation in girl with BCAP31-related syndrome
}

\author{
Hsiao-Jung Kao ${ }^{1}$, Hung-Lun Chiang ${ }^{1}$, Hsiao-Huei Chen ${ }^{1}$, Pi-Chuang Fan ${ }^{2}$, Yi-Fang Tu$^{3}$, \\ Yen-Yin $\mathrm{Chou}^{3}$, Wuh-Liang Hwu ${ }^{2}$, Chien-Ling Lin ${ }^{1}$, Pui-Yan Kwok ${ }^{1}$, and Ni-Chung Lee ${ }^{2}$ \\ ${ }^{1}$ Academia Sinica \\ ${ }^{2}$ National Taiwan University Hospital \\ ${ }^{3}$ National Cheng Kung University Hospital
}

May 6, 2020

\begin{abstract}
Full genome analysis of a young girl with deafness, dystonia, central hypomyelination, refractory seizure, and fluctuating liver function impairment revealed a heterozygous, de novo variant in the BCAP31 gene on chromosome X28q (NC_000023.11(BCAP31_v001):c.92G>A), mutations of which caused the X-linked recessive severe neurologic disorder DDCH (Deafness, Dystonia, and Cerebral Hypomyelination, OMIM\#300475). Reverse transcription-PCR (RT-PCR) of the patient's white blood cells showed the absence of wild-type BCAP31 mRNA but the presence of two novel BCAP31 mRNAs. The major alternatively-spliced mRNA is due to exon 2 skipping and the utilization of a new initiation site in exon 3 that leads to a frameshift and truncated transcript while the minor novel mRNA has a 110 nucleotide insertion to exon 2. Phasing studies showed that the de novo variant arose in the paternal $\mathrm{X}$ chromosome. X chromosome inactivation assay was done and confirmed that the patient's maternal X chromosome was preferentially inactivated, providing evidence that the mutated BCAP31 gene was the predominantly expressed. According to the ACMG guideline, this variant is deemed "pathogenic" (PS2, PS3, PM2, PP3, PP4) and deleterious. This is the first reported female patient in BCAP31-related syndrome resulted from skewed X-inactivation and a de novo mutation in the active $\mathrm{X}$ chromosome.
\end{abstract}

\section{Keywords}

BCAP31, X-inactivation, DDCH, splicing variants, female with X-linked recessive disease

\section{Introduction}

Developmental delay and congenital neurologic disorder are rare conditions caused by gene mutations that are hard to identify due to phenotypic and genetic heterogeneity. While whole exome sequencing (WES) has been successful in identifying some causal mutations, most cases remain unsolved. Since 2012, multiple groups have reported that males with hemizygous defect in BCAP31 gene on Xq28 involving splicing site mutations, nonsense or small indels result in severe phenotypes such as Deafness, Dystonia, And Cerebral Hypomyelination (DDCH, OMIM\#300475) or contiguous ABCD1/DXS1375E deletion syndrome (CADDS) (Albanyan, Al Teneiji, Monfared, \& Mercimek-Mahmutoglu, 2017; Cacciagli et al., 2013; Rinaldi, Van Hoof, Corveleyn, Van Cauter, \& de Ravel, 2020; Shimizu et al., 2020; Vittal, Hall, \& Berry-Kravis, 2015; Vittal, Hall, Dames, Mao, \& Berry-Kravis, 2016; Whalen et al., 2019). These phenotypes are also found with microdeletions between $S L C 6 A 8$ and $A B C D 1$ or partial deletion of $S L C 6 A 8-B C A P 31$ (Calhoun \& Raymond, 2014; Osaka et al., 2012; van de Kamp et al., 2015). BCAP31 encodes B-cell receptor-associated protein 31, with ubiquitous expression and is abundant in endoplasmic reticulum (ER) membrane. BCAP31 is found to be involved in the anterograde transport of ER-to-Golgi exchanges, in ER associated-degradation (ERAD) 
and in caspase 8-mediated apoptosis (Annaert, Becker, Kistner, Reth, \& Jahn, 1997; Nguyen, Breckenridge, Ducret, \& Shore, 2000; Wakana et al., 2008).

Among the reported cases involving BCAP31 mutations, only one derived from a de novo mutation (Rinaldi et al., 2020). Most male patients inherited mutated chromosome X (chrX) from non-symptomatic carrier mothers; however, in two of the reported cases, the patients' mothers presented mild features. In addition, one heterozygous mother has bilateral sensorineural hearing loss (Albanyan et al., 2017), and another heterozygous mother has similar facial features to her two affected sons, such as long face and ocular hypotelorism, but no neurological or cognitive symptoms (Vittal et al., 2016).

Female carriers of X-linked disorders such as DDCH are normally not affected because they carry a normal copy of the gene. However, to compensate for the dosage effect of different $\mathrm{X}$ chromosome numbers in male (XY) and female (XX), X chromosome inactivation (XCI) happens in female cells (Minks, Robinson, \& Brown, 2008). XCI is an epigenetic remodeling through random methylation of each parental chromosome at the 8-16 cell stage (Amos-Landgraf et al., 2006; Shvetsova et al., 2019; van den Berg et al., 2009). Once XCI is established in each cell, the imprinting XCI pattern is stably penetrated to the divided cells. Individuals who have balanced inactivation between paternal and maternal X chromosomes present 50:50 XCI in cell pools. However, some individuals are found to have an unbalanced XCI ratio, resulting in skewed $\mathrm{XCI}$, commonly defined as a ratio of inactivation to activation $>70 \%$, with preferential inactivation of one parental X chromosome. Theoretically, a female carrying a mutation in gene associated with an X-linked recessive disorder will be symptomatic if she has skewed XCI in which the X chromosome with the wild-type allele is preferentially inactivated.

In this report, we present evidence that the molecular defect of a young girl with deafness, dystonia, central hypomyelination, refractory seizure, and fluctuating liver function abnormalities is due to ade novo variant in BCAP31 arose in her paternal X chromosome and skewed X-inactivation of her maternal X chromosome. This is the first report of a female patient of BCAP31-related syndrome due to a pathogenic mutation in $B C A P 31$ in the context of skewed X-inactivation.

\section{Material and Methods}

\section{DNA extraction and full genome analysis pipeline}

Fresh peripheral blood samples were collected from the patient at age 4 and her two biological parents. After removing the red blood cells by RBC lysis buffer (Qiagen), the high-molecular-weight genomic DNA (HMW gDNA) was extracted from the white blood cells (WBCs) of each sample by a plug lysis process with the Bionano Prep kit (Bionano Genomics). HMW gDNA from the trio then underwent 10x Genomics (10xG) Linked-Reads Chromium whole genome sequencing (WGS) on the NovaSeq 6000 Sequencing System (Illumina) to 40-60X read depth and optical mapping on the Bionano Genomics Saphyr System to 60X coverage. The FASTQ files were processed and aligned with GRCh38 (hg38, Genome Reference Consortium) via Long Ranger BASIC and ALIGN Pipelines (10x Genomics) for linked-read alignment, variant calling, phasing, and structural variant Calling. Thede novo, phased genome assemblies of the patient and her two biological parents were compared to identify single nucleotide variants (SNVs) and structural variants (SVs). After filtering out synonymous SNVs and common SNVs/SVs based on data from the 1000 Genomes Project, Exome Aggregation Consortium (ExAC), and Genome Aggregation Database (gnomAD), the genes affected by SNVs, SVs, or a combination of SNVs and SVs were examined in detail. In consultation with the clinicians, a thorough literature search was done on candidate genes with variants (both SNVs and SVs) predicted as deleterious by multiple tools in the web-based wANNOVAR suite to look for genotype-phenotype correlation. All candidate variants were confirmed by Sanger sequencing of PCR products amplified from the individuals. The following primers were used for the BCAP31 mutation: forward 5'-AGCGAACGGAAGTTTGTG-3' and reverse 5'-GGTGGGAGGGCATCATTT-3'.

\section{$\mathrm{X}$ chromosome inactivation (XCI) assay}

The HUMARA (Human Androgen Receptor) assay (Allen, Zoghbi, Moseley, Rosenblatt, \& Belmont, 1992; 
Bertelsen, Tumer, \& Ravn, 2011; Echevarria et al., 2016) was used to determine the ratio of inactivated paternal vs maternal X chromosomes. Briefly, gDNA samples $(0.25 \mathrm{ug})$ were incubated with $10 \mathrm{U}$ HpaII (New England Biolabs) at $37^{\circ} \mathrm{C}$ overnight followed by heat inactivation of the enzyme at $80^{\circ} \mathrm{C}$ for $20 \mathrm{~min}$. The highly polymorphic androgen receptor promotor region of the HpaII treated samples and untreated control samples were amplified with $0.2 \mu \mathrm{M}$ forward primer 5'-FAM -TCCAGAATCTGTTCCAGAGCGTGC-3' and $0.2 \mu \mathrm{M}$ reverse primer 5'-TCCAGAATCTGTTCCAGAGCGTGC-3' with $0.2 \mathrm{mM} \mathrm{dNTP,} 1.5 \mathrm{mM} \mathrm{MgCl}_{2}$ and $0.025 \mathrm{U}$ AmpliTaq Gold DNA Polymerase (Thermo Fisher) by PCR amplification (initial denaturation at $95^{\circ} \mathrm{C}$ for $6 \mathrm{~min}$ followed by 35 cycles of $40 \mathrm{~s}$ at $95^{\circ} \mathrm{C}, 30 \mathrm{~s}$ at $60^{\circ} \mathrm{C}$, and $1 \mathrm{~min}$ at $72^{\circ} \mathrm{C}$ and a final extension step at $72^{\circ} \mathrm{C}$ for $2 \mathrm{~min}$ ). After adding the GeneScan 500 LIZ dye Size Standard (Thermo Fisher), the mixtures were analyzed on an ABI 3730 DNA Analyzer with GeneMapper software 3.7 (Applied Biosystems). XCI ratio was estimated by the area of the smaller base size $\left(\mathrm{XC}_{1}\right)$ and larger base size $\left(\mathrm{XC}_{2}\right)$ peaks, with formula as follows: peak area of $\left[\left(\mathrm{XC}_{1}{ }^{\text {digested }} / \mathrm{XC}_{1}{ }^{\text {nondigested }}\right) /\left(\left(\mathrm{XC}_{1}{ }^{\text {digested }} / \mathrm{XC}_{1}{ }^{\text {nondigested }}\right)+\left(\mathrm{XC}_{2}{ }^{\text {digested }} / \mathrm{XC}_{2}{ }^{\text {nondigested }}\right)\right)\right]$.

\section{RNA extraction and RT-PCR}

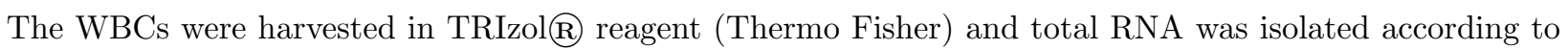
the manufacturer's instructions. cDNA was prepared from $1 \mu \mathrm{g}$ total RNA, using the SuperScript@VI reverse transcriptase with random hexamers, following the manufacturer's protocol (Thermo Fisher). RT-PCR was done using the primer pair of BCAP31ex1F 5'-CACGTTGACTGTGGGAAACTC-3' and BCAP31ex4R 5'TTCCGTCACATCATCATACTTCC-3'. All RT-PCR products were gel extracted and sequenced to confirm the normal splicing, intron inclusion, and exon skipping forms.

\section{Ethical Compliance}

Institutional ethical review and approval was granted (IRB No.AS-IRB01-19041(N)), and informed consent was provided, for all data obtained from patients.

\section{Results}

\section{Clinical features of the patient}

The female subject was the $2^{\text {nd }}$ child of the family born at 38 weeks with a birth body weight of $2990 \mathrm{~g}$. There were no remarkable perinatal events or maternal history. Bilateral deafness, hypothyroidism and ventricular septal defects type II were noted in the neonatal period. Episodic dyskinetic movements began at 6 months of age that progressed to general dystonia by 11 months. These dyskinetic/dystonia movements presented as eye deviation and head deviation to the left associated with tongue protrusion, tonic movements of bilateral upper and lower extremities lasting 3 to 60 seconds per episode, with frequency of up to 40 episodes per hour. Brain magnetic resonance imaging (MRI) done on several occasions showed mild cerebral atrophy with bilateral ventricular dilatation, thinning of corpus callosum, decreased subcortical and periventricular white matter and delayed myelination (Figure 1). Electroencephalogram (EEG) done on several occasions were remarkable for global cortical dysfunction and paroxysmal discharges mainly over left hemisphere. Concurrent problems included profound developmental delay, failure to thrive, intractable focal to bilateral tonic-clonic seizures, fluctuating liver function impairment (ALT/AST 227/148 U/L), and recurrent aspiration pneumonia. There was no elevation of creatine kinase $(296 \mathrm{U} / \mathrm{L})$ or lactate $(1.26 \mathrm{mM})$. Muscle biopsy only showed neurogenic myopathy with normal electron transfer chain activity. $P O L G$ mutation analysis, tandem mass of metabolites, dried blood spot 3-O-methyldopa level were negative. She had progressive deterioration with febrile episodes and passed away at 5 years old. Exome sequencing by an outside group performed previously using a candidate gene panel analysis approach for intellectual disability (that did not include the BCAP31 gene), followed by whole exome analysis based on the phenotypes of intellectual disability, epilepsy, hypotonia, and MRI abnormalities, did not reveal any candidate variants.

\section{Identification of a de novo variant in BCAP31 by full genome analysis}

Since exome sequencing did not reveal candidate variants in the primary screen, we performed Chromium (10x Genomics) Linked-Reads whole genome sequencing on the patient and her biological parents. All variants were called and phased with Long Ranger BASIC and ALIGN Pipelines and followed by filters 
of allelic frequency with threshold set at 0.01 for missense, indels and splice site mutations. Then, the remaining variants after filtering were evaluated according to three possible genetic models: autosomal recessive, autosomal dominant and $\mathrm{X}$-linked recessive. As the parents were without the disorder, only de novo variants were considered in the autosomal dominant and X-linked recessive models. After determining that the sequence quality was high by expert inspection, and literature review that showed good correlation with the associated clinical phenotype, we identified a de novoheterozygous variant in the BCAP31 gene as a prime candidate for the patient's condition. This mutation is confirmed by Sanger DNA sequencing and is found on the plus strand of hg38 chrX:153723153C > T that predicts to cause NM_001256447.2:c.92G>A, p.Arg31Lys change (Fig. 2). This novel variant has not been reported in Taiwan BioBank or any other database. Even with careful analysis of the sequencing and optical mapping data, no other disease-causing variant (including SNVs, small indels, and structural variation) could be identified in the patient'sBCAP31 gene.

\section{Novel BCAP31 alternative splicing transcripts in patient}

The novel variant (NM_001256447.2:c.92G $>$ A) sits at the $2^{\text {nd }}$ anticodon that encodes the $31^{\text {th }}$ amino acid of BCAP31 which is highly conserved in vertebrates. Moreover, the variant is part of a consensus 5' donor splice site sequence at the end of the exon. We evaluated the wildtype and variant allele sequence by the Human Splicing Finder (HSF) (Desmet et al., 2009) to see whether the variant would cause any changes to the splice site. The HSF predicts that the wild type $\mathrm{G}$ allele position is a donor splice recognition site (underlined G in CTCCTAAAA $G$ gtatggccta), but that the mutant A allele will alter the donor site recognition, thereby disrupting proper splicing.

To confirm the hypothesis that the A variant in the patient'sBCAP31 gene would lead to improper splicing, we examined the transcript pattern of white blood cells (WBCs) in the patient and her parents by RT-PCR followed by Sanger sequencing (Fig 3). The results showed that there were no wildtype transcript in the patient's WBCs, but that two novel transcripts were found. The major novel transcript is devoid of exon 2 (NM_001256447.2:r.54_190del, p.Ser2AlafsTer42) while the minor transcript has a 110nt insertion in intron 2 (NM_001256447.2:r.190_191ins[g>a;190+1_190+110], p.Arg31LysfsTer11). The major transcript leads to a frameshift transcript utilizing a new start codon site in exon 3.

In summary, a de novo heterozygous variant in the patient results in a novel frameshift transcript that replaces the wildtype form of BCAP31. This variant is deemed Pathogenic (ACMG criteria PS2, PS3, PM2, PP3, PP4), according to de novo variant (PS2), RT-PCR study (PS3), absent from controls (PM2), deleterious effect on the gene product (PP3), and clinical phenotype (PP4) based on the criteria of ACMG/AMP 2015 guideline (Li \& Wang, 2017).

\section{Skewed $\mathrm{X}$ chromosome inactivation in the patient}

Because the patient was a girl, we expected that she would express both wildtype and mutant transcripts in her tissues. However, she only expressed the defective frameshift BCAP31 transcript and her clinical features resembled those of X-linked recessive male patients carrying BCAP31 mutations. Haplotype phasing with linked-read sequencing data around the BCAP31 gene showed that the de novo allele arose from the paternal $\mathrm{X}$ chromosome while the wild type allele came from the maternal X chromosome (Figure 4). To explain the patient's BCAP31 expression pattern and clinical features, we hypothesized that uneven X chromosome inactivation (XCI) occurred in the patient. We performed the HUMARA (Human Androgen Receptor Amplification) assay to assess the methylation ratio of maternal and paternal X chromosome in WBCs, with the knowledge that XCI patterns between blood and other analyzed tissues were highly correlated (Echevarria et al., 2016). The androgen receptor allele profile of triplicate experiments showed skewed X chromosome inactivation, with the maternal X chromosome (carrying the wildtype BCAP31 allele) inactivated in $88 \%+-$ $2 \%$ of the WBCs (Figure 5). The HUMARA study confirms that the paternal X chromosome (carrying the de novo mutation) was active in the majority of the cells and explained the patient's "X-linked recessive" phenotype.

\section{Discussion}


The molecular diagnosis of rare congenital disorders has changed from family studies by linkage analysis followed by positional cloning to direct whole exome or whole genome next generation sequencing. Because every person in the world carries thousands of gene mutations, a diagnosis can only be made when a mutation is consistent with both the genetic mode of inheritance and the functional changes leading to the disorder. In these "experiments of one", reliance of previously published cases of similar clinical features and gene mutations makes it challenging to diagnose cases where mutations are found in genes the functions of which are little known. Similarly, it is difficult to make a diagnosis when a deleterious mutation is found in only one copy of a gene that causes an autosomal recessive disorder.

This study illustrates a way to establish a molecular diagnosis when there are issues with the mode of inheritance or functional consequences of a candidate gene mutation. By conducting additional experiments beyond whole exome or whole genome sequencing, one can determine the functional consequence of a mutation and explain the inheritance pattern of the condition. In our case, while the BCAP31 gene is well established as one associated with DDCH and our patient has clinical features of DDCH, the particular heterozygous de novo mutation in our patient has not been reported before. Furthermore, her case is not consistent with the X-linked recessive mode of inheritance.

We conducted 3 sets of experiments to characterize our case. First, we exhaustively searched for mutations in the maternally inherited $B C A P 31$ gene by linked-read whole genome sequencing and optical mapping to determine that the maternally inherited $B C A P 31$ region harbors no deleterious mutations. Second, we conducted an XCI assay to confirm the hypothesis that preferential X inactivation of the maternally inherited $\mathrm{X}$ chromosome bearing the wild-type $B C A P 31$ gene created a setting where the patient expresses predominately the mutated BCAP31 gene. Third, we conducted RNA studies to show that our patient expresses predominately the mutated gene, resulting in a frameshifted transcript that skipped over exon 2.

XCI status has been used to explain the wide spectrum of phenotypes observed (from asymptomatic to severe) in some X-linked diseases in women, depending on the degree of silencing of the normal allele (Echevarria et al., 2016; Elstein, Schachamorov, Beeri, \& Altarescu, 2012; Fahim et al., 2019; Juchniewicz et al., 2018). For example, the severity scores and progression phenomena in X-linked Fabry disease caused by $\alpha$-Gal deficiency had significant correlation with XCI ratio in female patients (Echevarria et al., 2016; Elstein et al., 2012; Juchniewicz et al., 2018). Given the recent observation that skewed XCI is common in the general female population (Shvetsova et al., 2019), female patients with clinical features of an X-linked recessive disorder and one copy of the mutated gene should be screened for skewed XCI.

BCAP31 has 2 protein isoforms translated from four transcripts that differ in the 5 ' UTR in exon 1 . Three transcripts (NM_001139441.1, NM_001256447.2, NM_005745.7) utilize the start codon in exon 2 and encode a canonical BCAP31 isoform 1 (UniPortKB ID: P51572-1). The NM_001139457.2 transcript utilizes the start codon in exon 1 and encodes a BCAP31 isoform 2 (UniPortKB ID: P51572-2). Exon 2 is conserved in all four transcripts. In our patient, the de novo mutation at the splicing donor site disrupted the mRNA structure by splicing out the entire exon 2, resulting in a frameshift transcript utilizing a new start codon site in exon 3. Consequently, the mutant protein is shortened and has a completely different structure, essentially rendering our patient a loss-of-function BCAP31 null. Full genome analysis that combines linked-read sequencing and optical mapping for comprehensive single nucleotide and structural variant identification with phasing allows one to characterize a de novo heterozygous deleterious mutation in a female patient with BCAP31-related syndrome. After determining that the de novo mutation arose in the paternal $\mathrm{X}$ chromosome and that the maternal $\mathrm{X}$ chromosome is preferentially inactivation, we conclude that the BCAP31 mutation is the cause of the patient's disorder because RNA studies confirms that her WBCs expresses only the mutant transcript. According to the ACMG guideline, this variant is deemed "pathogenic" (PS2, PS3, PM2, PP3, PP4) and deleterious. Our study illustrates that the combination of full genome analysis (for comprehensive variant detection and phasing), RNA studies (for alternative splicing and transcript analysis), and XCI studies for female patients with features of X-linked recessive disorders can lead to molecular diagnosis of difficult cases of monogenic disorders.

\section{Data Availability}


The data that support the findings of this study are available from the corresponding author upon reasonable request.

\section{Acknowledgement}

We thank the patient's family for their participation in this study and the National Center for Genome Medicine for technical support.

\section{References:}

Albanyan, S., Al Teneiji, A., Monfared, N., \& Mercimek-Mahmutoglu, S. (2017). BCAP31-associated encephalopathy and complex movement disorder mimicking mitochondrial encephalopathy. Am J Med Genet A, 173 (6), 1640-1643. doi:10.1002/ajmg.a.38127

Allen, R. C., Zoghbi, H. Y., Moseley, A. B., Rosenblatt, H. M., \& Belmont, J. W. (1992). Methylation of HpaII and HhaI sites near the polymorphic CAG repeat in the human androgen-receptor gene correlates with X chromosome inactivation. Am J Hum Genet, 51 (6), 1229-1239. Retrieved from https://www.ncbi.nlm.nih.gov/pubmed/1281384

https://www.ncbi.nlm.nih.gov/pmc/articles/PMC1682906/pdf/ajhg00070-0056.pdf

Amos-Landgraf, J. M., Cottle, A., Plenge, R. M., Friez, M., Schwartz, C. E., Longshore, J., \& Willard, H. F. (2006). X chromosome-inactivation patterns of 1,005 phenotypically unaffected females. Am J Hum Genet, 79 (3), 493-499. doi:10.1086/507565

Annaert, W. G., Becker, B., Kistner, U., Reth, M., \& Jahn, R. (1997). Export of cellubrevin from the endoplasmic reticulum is controlled by BAP31. J Cell Biol, 139 (6), 1397-1410. doi:10.1083/jcb.139.6.1397

Bertelsen, B., Tumer, Z., \& Ravn, K. (2011). Three new loci for determining x chromosome inactivation patterns. J Mol Diagn, 13 (5), 537-540. doi:10.1016/j.jmoldx.2011.05.003

Cacciagli, P., Sutera-Sardo, J., Borges-Correia, A., Roux, J. C., Dorboz, I., Desvignes, J. P., . . . Villard, L. (2013). Mutations in BCAP31 cause a severe X-linked phenotype with deafness, dystonia, and central hypomyelination and disorganize the Golgi apparatus. Am J Hum Genet, 93 (3), $579-586$. doi:10.1016/j.ajhg.2013.07.023

Calhoun, A. R., \& Raymond, G. V. (2014). Distal Xq28 microdeletions: clarification of the spectrum of contiguous gene deletions involving ABCD1, BCAP31, and SLC6A8 with a new case and review of the literature.Am J Med Genet A, 164A (10), 2613-2617. doi:10.1002/ajmg.a.36661

Desmet, F. O., Hamroun, D., Lalande, M., Collod-Beroud, G., Claustres, M., \& Beroud, C. (2009). Human Splicing Finder: an online bioinformatics tool to predict splicing signals. Nucleic Acids Res, 37 (9), e67. doi:10.1093/nar/gkp215

Echevarria, L., Benistan, K., Toussaint, A., Dubourg, O., Hagege, A. A., Eladari, D., . . . Germain, D. P. (2016). X-chromosome inactivation in female patients with Fabry disease. Clin Genet, 89 (1), $44-54$. doi:10.1111/cge. 12613

Elstein, D., Schachamorov, E., Beeri, R., \& Altarescu, G. (2012). X-inactivation in Fabry disease. Gene, 505 (2), 266-268. doi:10.1016/j.gene.2012.06.013

Fahim, A. T., Sullivan, L. S., Bowne, S. J., Webb-Jones, K., Wheaton, D. K. H., Branham, K. E., . . . Daiger, S. P. (2019). X-Chromosome Inactivation is a Biomarker of Clinical Severity in Female Carriers of $\mathrm{X}$-linked Retinitis Pigmentosa. Investigative Ophthalmology 6 Visual Science, 60 (9). Retrieved from <Go to ISI>://WOS:000488628107093

Juchniewicz, P., Kloska, A., Tylki-Szymanska, A., Jakobkiewicz-Banecka, J., Wegrzyn, G., Moskot, M., . . . Piotrowska, E. (2018). Female Fabry disease patients and X-chromosome inactivation. Gene, 641 , $259-264$. doi:10.1016/j.gene.2017.10.064 
Li, Q., \& Wang, K. (2017). InterVar: Clinical Interpretation of Genetic Variants by the 2015 ACMG-AMP Guidelines. American Journal of Human Genetics, 100 (2), 267-280. doi:10.1016/j.ajhg.2017.01.004

Minks, J., Robinson, W. P., \& Brown, C. J. (2008). A skewed view of X chromosome inactivation. J Clin Invest, 118 (1), 20-23. doi:10.1172/JCI34470

Morrone, A., Cavicchi, C., Bardelli, T., Antuzzi, D., Parini, R., Di Rocco, M., . . . Zammarchi, E. (2003). Fabry disease: molecular studies in Italian patients and X inactivation analysis in manifesting carriers. $J$ Med Genet, 40 (8), e103. doi:10.1136/jmg.40.8.e103

Nguyen, M., Breckenridge, D. G., Ducret, A., \& Shore, G. C. (2000). Caspase-resistant BAP31 inhibits fas-mediated apoptotic membrane fragmentation and release of cytochrome c from mitochondria. Mol Cell Biol, 20 (18), 6731-6740. doi:10.1128/mcb.20.18.6731-6740.2000

Osaka, H., Takagi, A., Tsuyusaki, Y., Wada, T., Iai, M., Yamashita, S., . . . Matsumoto, N. (2012). Contiguous deletion of SLC6A8 and BAP31 in a patient with severe dystonia and sensorineural deafness. Mol Genet Metab, 106 (1), 43-47. doi:10.1016/j.ymgme.2012.02.018

Rinaldi, B., Van Hoof, E., Corveleyn, A., Van Cauter, A., \& de Ravel, T. (2020). BCAP31-related syndrome: The first de novo report. Eur J Med Genet, 63 (2), 103732. doi:10.1016/j.ejmg.2019.103732

Shimizu, K., Oba, D., Nambu, R., Tanaka, M., Oguma, E., Murayama, K., . . . Ohashi, H. (2020). Possible mitochondrial dysfunction in a patient with deafness, dystonia, and cerebral hypomyelination (DDCH) due to BCAP31 Mutation. Mol Genet Genomic Med, 8 (3), e1129. doi:10.1002/mgg3.1129

Shvetsova, E., Sofronova, A., Monajemi, R., Gagalova, K., Draisma, H. H. M., White, S. J., . . Consortium, G. (2019). Skewed X-inactivation is common in the general female population. European Journal of Human Genetics, 27 (3), 455-465. doi:10.1038/s41431-018-0291-3

van de Kamp, J. M., Errami, A., Howidi, M., Anselm, I., Winter, S., Phalin-Roque, J., . . Salomons, G. S. (2015). Genotype-phenotype correlation of contiguous gene deletions of SLC6A8, BCAP31 and ABCD1.Clin Genet, 87 (2), 141-147. doi:10.1111/cge.12355

van den Berg, I. M., Laven, J. S. E., Stevens, M., Jonkers, I., Galjaard, R. J., Gribnau, J., \& van Doorninck, J. H. (2009). X Chromosome Inactivation Is Initiated in Human Preimplantation Embryos.American Journal of Human Genetics, 84 (6), 771-779. doi:10.1016/j.ajhg.2009.05.003

Vittal, P., Hall, D. A., \& Berry-Kravis, E. (2015). BCAP31 mutation causing congenital dystonia and central hypomyelination discovered using exome sequencing. Movement Disorders, 30 , S206-S207. Retrieved from $<$ Go to ISI >://WOS:000356598200524

Vittal, P., Hall, D. A., Dames, S., Mao, R., \& Berry-Kravis, E. (2016). BCAP31 Mutation Causing a Syndrome of Congenital Dystonia, Facial Dysorphism and Central Hypomyelination Discovered Using Exome Sequencing. Mov Disord Clin Pract, 3 (2), 197-199. doi:10.1002/mdc3.12250

Wakana, Y., Takai, S., Nakajima, K., Tani, K., Yamamoto, A., Watson, P., . . . Tagaya, M. (2008). Bap31 is an itinerant protein that moves between the peripheral endoplasmic reticulum (ER) and a juxtanuclear compartment related to ER-associated Degradation. Mol Biol Cell, 19 (5), 1825-1836. doi:10.1091/mbc.E0708-0781

Whalen, S., Gomart, A., Mignot, C., De Villemeur, T. B., Gerasimenko, A., Heron, D., . . . ChantotBastaraud, S. (2019). Novel patient with Contiguous ABCD1/DXS1375E (BCAP31) Deletion Syndrome (CADDS) and review of the literature. European Journal of Human Genetics, 27, 1497-1498. Retrieved from < Go to ISI >://WOS:000489313903213 

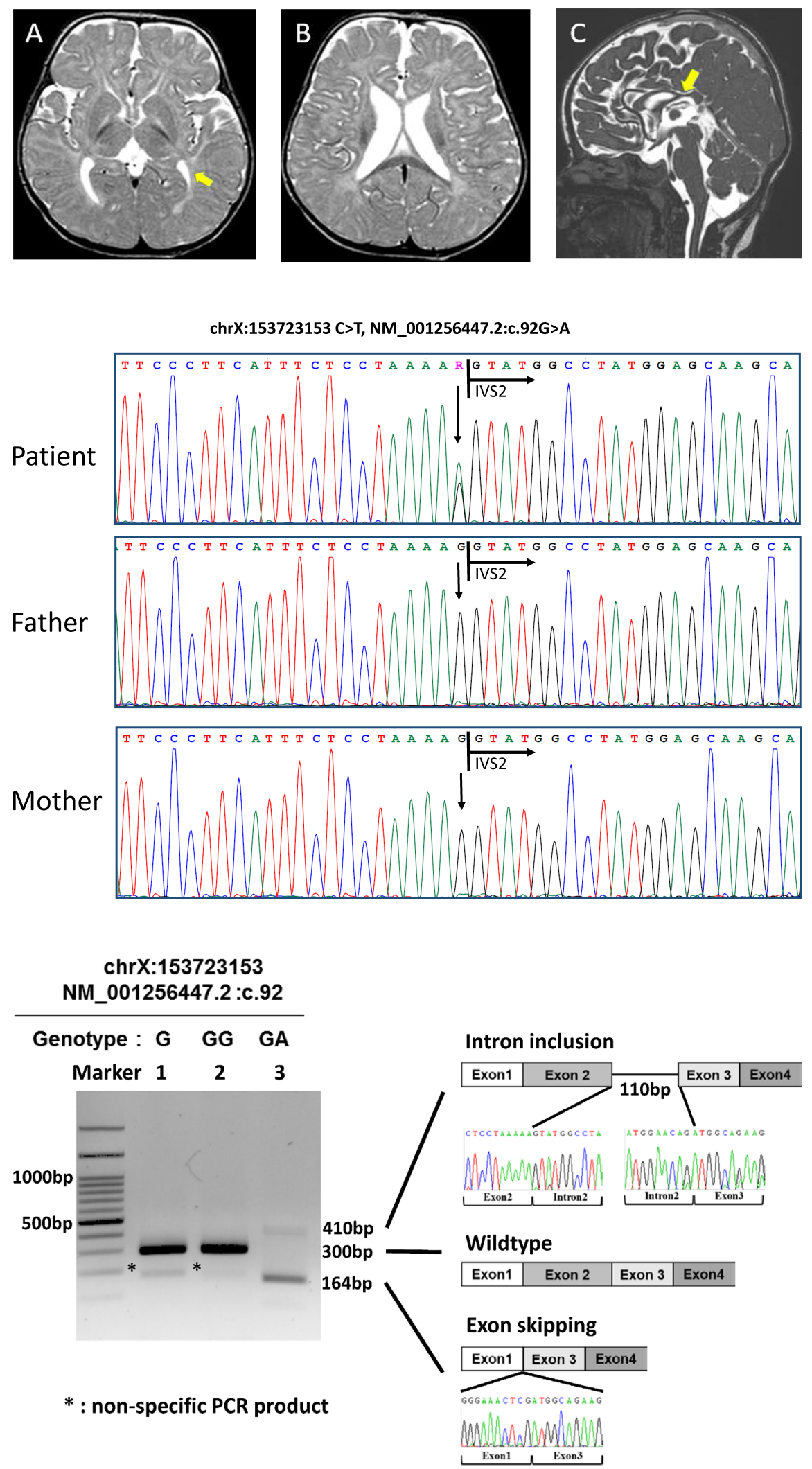


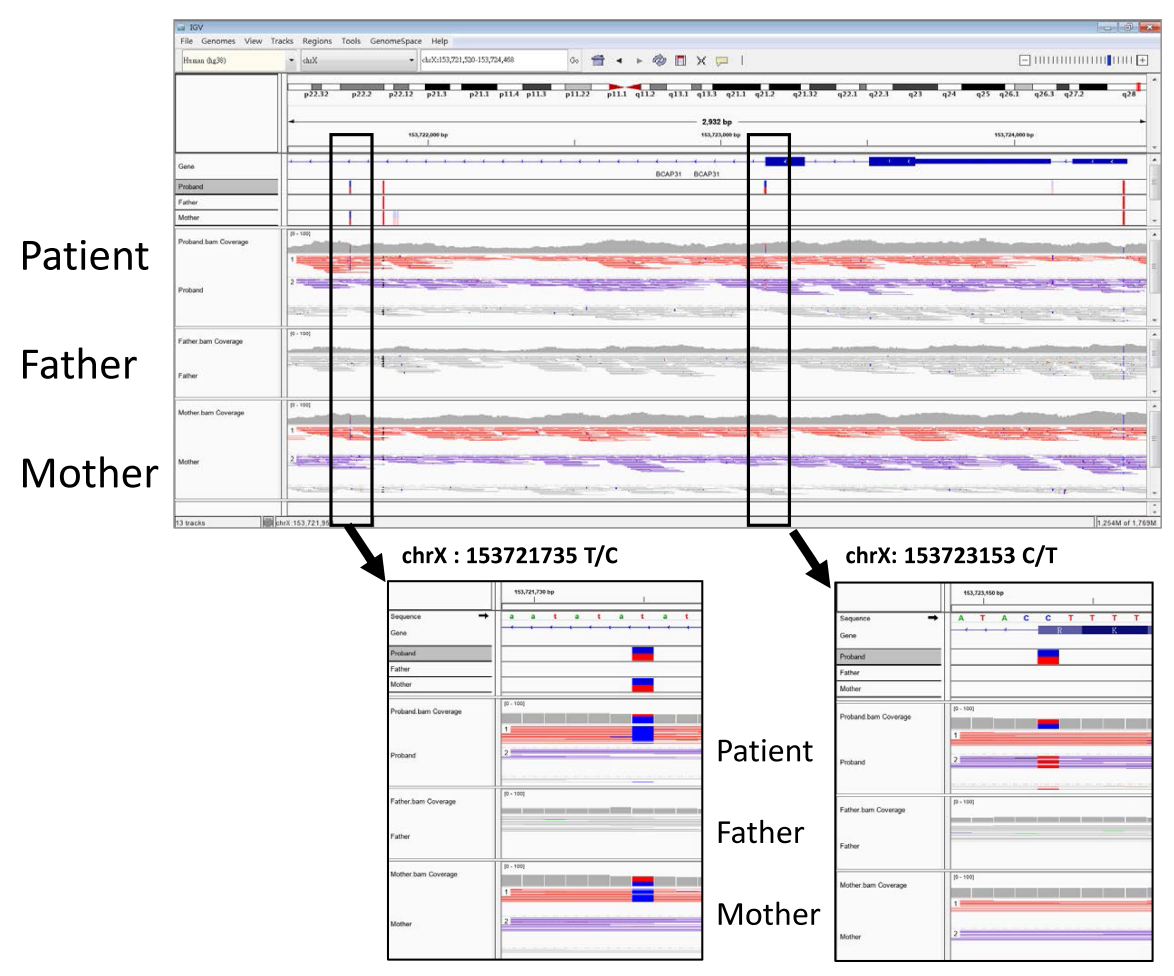

(a)

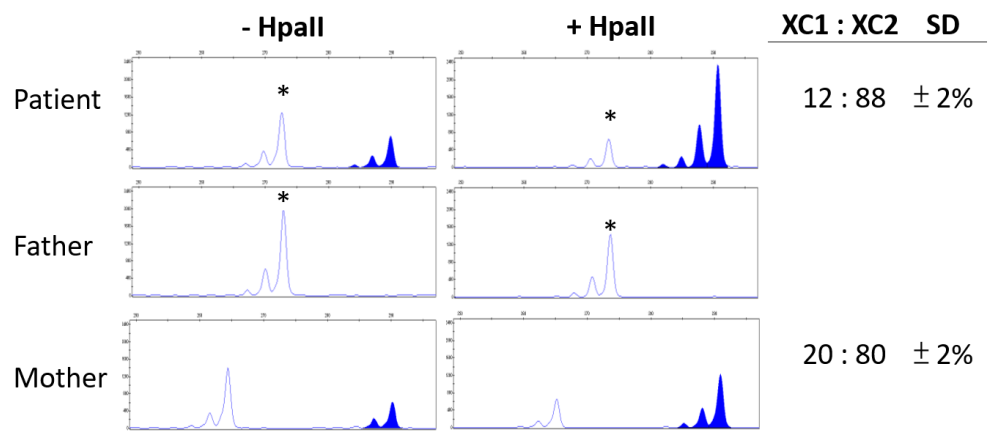

(b)

XCI Ratio $=\frac{\text { XC1 DIGESTION } / \text { XC1 UNDIGESTION }}{(\text { XC1 DIGESTION/XC1 UNDIGESTION })+(\text { XC2 DIGESTION/XC2 UNDIGESTION })}$ 